\title{
Phosphorylation of the 2,2'-Dihydroxy-1,1'-dinaphthylmethane and Synthesis of Phosphamacrocycles on its Basis
}

\author{
Pavel V. Slitikov, ${ }^{a}$ and Elena N. Rasadkina ${ }^{\mathrm{b}}$ \\ aBauman Moscow State Technical University, 105005 Moscow, Russian Federation \\ ${ }^{\mathrm{b}}$ Moscow Pedagogical State University, Institute of Biology and Chemistry, 119021 Moscow, Russian Federation \\ ${ }^{\circledR}$ Corresponding authorE-mail: pavlasiy@mail.ru
}

\begin{abstract}
Phosphorylation of 2,2'-dihydroxy-1,1'-dinaphthylmethane with diamidophosphites with different substituents at phosphorus atom was investigated. It was demonstrated that leaving of either two amide groups or phenyl group occurs upon formation of 1,3,2-dioxaphosphacine. Phosphamacrocyclic systems containing fragments of 2,2'-dihydroxy1,1'-dinaphthylmethane and aromatic diols - resorcinol and 1,3-dihydroxynaphthalene - were synthesized, their low stability in solution is specified. Oxidation reactions of the synthesized compounds were studied.
\end{abstract}

Keywords: Phosphorylation, 2,2'-dihydroxy-1,1'-dinaphthylmethane, diamidophosphites, aromatic diols, molecular assembly, NMR spectroscopy, X-ray.

\section{Фосфорилирование 2,2'-Аигидрокси-1,1'-Аинафтилметана и синтез фосфомакроциклов на его основе}

\author{
П. В. Слитиков, ${ }^{a}$ Е. Н. Расадкина ${ }^{\mathrm{b}}$

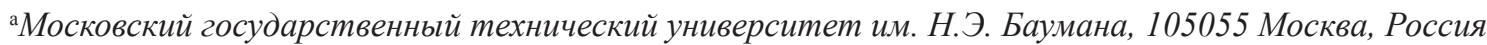 \\ 'Московский педагогический государственный университет, Институт биологии и химии, 119021 Москва, Россия \\ ${ }^{\circledR}$ E-mail: pavlasiy@mail.ru
}

\begin{abstract}
Проведено фосфорилирование 2,2'-дигидрокси-1,1'-динафтилметана диамидоэфирами фосфористой кислоть с различными заместителями у атома фосфора; показана принципиальная возможность ухода как двух амидных групп, так и фенильного заместителя при образовании 1,3,2-диоксафосфацина. Синтезированы фосфомакрочиклические системы, содержащие в своей структуре остатки 2,2'-дигидрокси-1,1'-динафтилметана и ароматических диолов - резориина и 1,3-дигидроксинафталина, отмечена их низкая устойчивость в растворах. Рассмотрены окислительные реакиии синтезированных систем.
\end{abstract}

Ключевые слова: Фосфорилирование, 2,2'-дигидрокси-1,1'-динафтилметан, диамидофосфиты, ароматические диолы, молекулярная сборка, спектроскопия ЯМР, рентгеноструктурный анализ. 


\section{Introduction}

Active studies of phosphorylation of naphthalene derivatives with two distal hydroxy-groups with di- and triamides of phosphorus acid were performed over last decade. ${ }^{[1-6]}$ As a result, there were synthesized macrocyclic compounds containing several fragments of macrocyclic diols and derivatives of phosphorous and phosphoric acids, which can be referred either as phosphacyclophanes or as benzocrown ethers. ${ }^{[7]}$ Such compounds can be used in synthesis of polynuclear complexes with transition metals for catalytic purposes, as well as supramolecular receptors which can capture ions and small molecules, and for structural tasks of modern organoelemental chemistry.

2,2'-Dihydroxy-1,1'-dinaphthylmethane 1 was investigated in reactions with derivatives of trivalent phosphorus together with other diatomic phenols. It was shown that application of alkyleneamidophosphites and other cyclic derivatives of $\mathrm{P}^{\mathrm{III}}$ has afforded open-chain molecules, which have been used as ligands for asymmetric catalysis. ${ }^{\left[{ }^{[8,9}\right.}$ Phosphorylation of $\mathbf{1}$ with triamidophosphites and other acyclic derivatives have resulted in formation of cyclic products 1,3,2-dioxaphophacines. ${ }^{[10-12]}$ At the same time, compound 1 has never been applied as a building block in synthesis of macrocyclic systems.

Therefore, the aim of the present investigation was to study applicability of $\mathbf{1}$ in synthesis of phosphamacrocycles.

\section{Results and Discussion}

At the first step, hexabutyltriamidophosphine (HBTA) 2a and trimorpholinophosphite (TMF) $\mathbf{2 b}$ were used as phosphorylating agents due to their low rate of phosphorylation in 1,4-dioxane in comparison with other triamides of phosphorous acid. ${ }^{[13]}$ However, even at reagent ratio 1:2a, $\mathbf{b}=1: 3$ the formation of bisamidophosphites was not observed in ${ }^{31} \mathrm{P}$ NMR spectra of reaction mixtures. Instead of them, in both cases 1,3,2-dioxaphosphacines $\mathbf{3} \mathbf{a}$ and $\mathbf{3 b}$ were obtained $\left(\delta_{\mathrm{p}}=140.1\right.$ and $134.3 \mathrm{ppm}$ respectively) (Scheme 1). Therefore, even application of phosphorylating agents $\mathbf{2} \mathbf{a}, \mathbf{b}$ with bulky substituents did not result in formation of bisphosophorylated derivatives.

At the next step, other phosphorylating agents were applied, namely, butyl and phenyl esters of phosphorous acid tetraethyldiamide $\mathbf{4 a , b}$ as well as phenyl ester of phospho- rous acid tetrabutyldiamide 4c. Phosporylation of 1 with these reagents was performed in acetonitrile and 1,4-dioxane (Scheme 2).

In the case of 4a irrespectively on reagent ratio (1:1, 1:2 or 1:3) in ${ }^{31} \mathrm{P}$ NMR spectra there was observed a singlet with c $\delta_{\mathrm{P}}=147.6 \mathrm{ppm}$ corresponding to 1,3,2-dioxaphosphacine 5a with ester substituent at $\mathrm{P}^{\mathrm{III}}$ atom. Notably, neither phosphacine $\mathbf{3}$ or bisphosphorylated system $\mathbf{5}$ were not formed. Therefore, it can be concluded that upon phosporylation of $\mathbf{1}$ by diamidophosphite containing aliphatic ester fragment, the leaving of amide groups is favourable. The rate of phosphorylation increases when passing from 1,4-dioxane to acetonitrile, which is common for this type of reactions.

Again, irrespectively on reagent ratio the reaction of bisnaphthol 1 with tetraethyldiamidophenylphosphite $\mathbf{4 b}$ has yielded 1,3,2-dioxaphosphacines $\mathbf{3 c}$ and $\mathbf{5 b}$ together with $b i$ sphosporylated derivatives $\mathbf{6 b}$ (Scheme 2). The latter compounds were found to be unstable and they gradually have converted into mixture of $\mathbf{3 c}$ and $\mathbf{5 b}$. Apparently, this process occurs via intramolecular dismutation, ${ }^{[14,15]}$ which leads to thermodynamically favourable 8 -membered ring system (Scheme 3).

The rate of this cyclization is faster in acetonitrile, while in 1,4-dioxane bisphosphorylated derivatives $\mathbf{6 b}, \mathbf{c}$ are more stable (up to 3 weeks).

Noteworthy, in contrast to diamidoester $\mathbf{4 a}$, in the case of $4 \mathbf{b}$ leaving of amide or ester fragments upon phosphorylation is equiprobable independently on reagent ratio as evidenced by ${ }^{31} \mathrm{P}$ NMR spectroscopy. Upon phosphorylation of bisnaphthol 1 with tetraethyldiamidophenylphosphite $\mathbf{4 b}$ in 1,4-dioxane or reaction mixture in one day after beginning in NMR spectrum there were observed resonance signals $\delta_{\mathrm{p}}=133.8(\mathbf{6 b})$ and $132.9 \mathrm{ppm}(\mathbf{4 b})$, while in one week there were observed resonance signals $\delta_{\mathrm{P}}=142.0(\mathbf{3 c}), 140.6(\mathbf{5 b})$ and $132.9 \mathrm{ppm}(\mathbf{4 b})$ with integral ratios 1:1:2.

An application of acetonitrile instead of 1,4-dioxane has resulted in increase of reaction rate ${ }^{[16]}-$ in two hours after beginning in ${ }^{31} \mathrm{P}$ NMR spectrum of reaction mixture there was observed only $\mathbf{6 b}$, however its signal has vanished in NMR spectrum afterwards.

In the case of tetrabutyldiamidophenylphosphite $\mathbf{4 c}$ phosphorylation of 1 was very slow. Even after 1 day in ${ }^{31} \mathrm{P}$ NMR spectrum the most intensive signal was $\delta_{\mathrm{P}}=132.5 \mathrm{ppm}$ corresponded to starting diamide $\mathbf{4 c}$, minor signal with $\delta_{\mathrm{P}}=142.6 \mathrm{ppm}$ corresponded to bisphosphorylated product 6c (Scheme 2). The latter have remained in reaction mix-
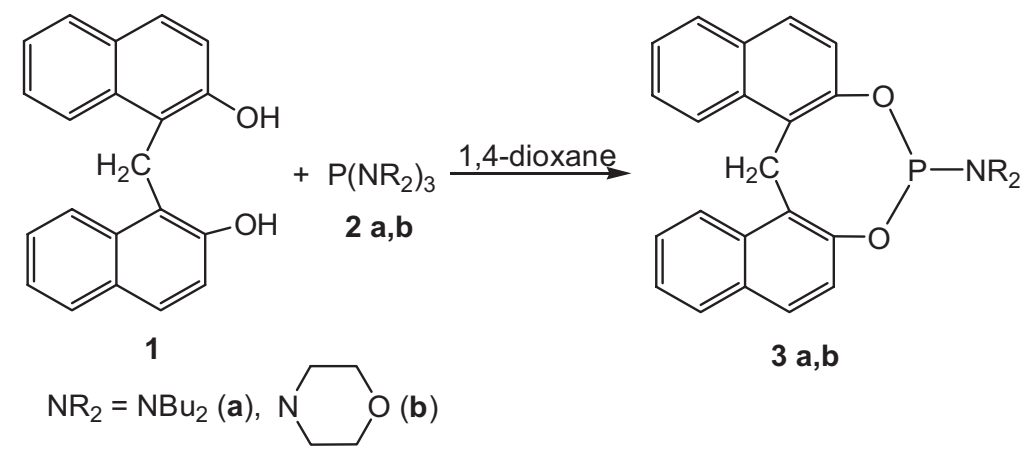

Scheme 1. Synthesis of 1,3,2-dioxaphosphacines 3a,b. 


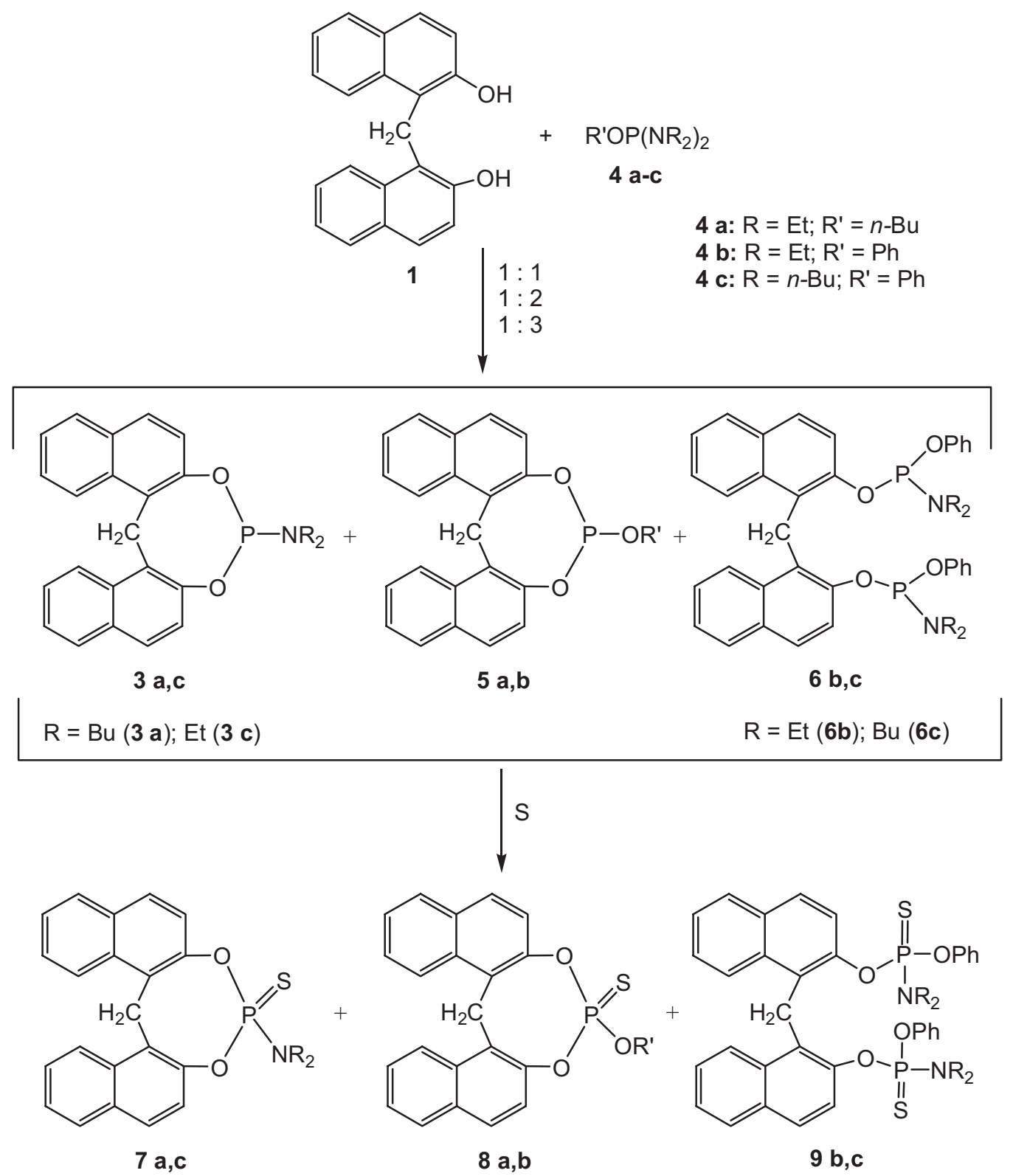

Scheme 2.<smiles>CC=NP(Oc1ccc2ccccc2c1Cc1c(OP(Oc2ccccc2)N(CC)c2ccccc2)ccc2ccccc12)N(CC)c1ccccc1</smiles>

$6 \mathrm{~b}$

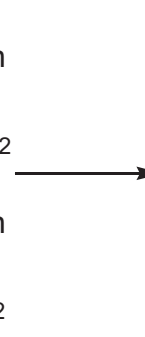<smiles>CCN1Oc2ccc3ccccc3c2Cc2c(ccc3ccccc23)O1</smiles>

$3 \mathrm{c}$<smiles>c1ccc(P(OPc2ccc3ccccc3c2Cc2cccc3ccccc23)c2ccccc2)cc1</smiles>

5 b

Scheme 3. 
ture even after 2 weeks after the beginning of reaction, together with 5b $\left(\delta_{\mathrm{p}}=141.8 \mathrm{ppm}\right), \mathbf{3 a}\left(\delta_{\mathrm{P}}=140.5 \mathrm{ppm}\right)$ and $4 \mathbf{c}\left(\delta_{\mathrm{p}}=132.5 \mathrm{ppm}\right)$. The integral ratios were 1.5:1:1:6. The slow reaction rate can be explained by lower reactivity of $\mathbf{4 c}$.

With the aim to separate and identify phosphorylated products, the reaction mixtures were sulfurized and column chromatography was used to isolate 1,3,2-dioxaphosphacines $\mathbf{7 a}, \mathbf{c}$ and $\mathbf{8 a}, \mathbf{b}$ and bisphosphorylated thione derivatives $\mathbf{9 b , c}$. Because of similarity of solubility and chromatographic mobility, isolation of compounds in pure forms was tedious, so the yields of individual thiones were modest. Physicalchemical properties of $\mathbf{7 c}$ have coincided with the previously reported data. ${ }^{[17]}$

X-Ray diffraction analysis of crystalline 8a was performed (Figure 1, Table 1). It has evidenced that the macrocyclic part of the molecule adopts bath-bath conformation. ${ }^{[17]}$ Stability of this conformation can be tentatively explained by the presence of bulky butoxy-substituent.

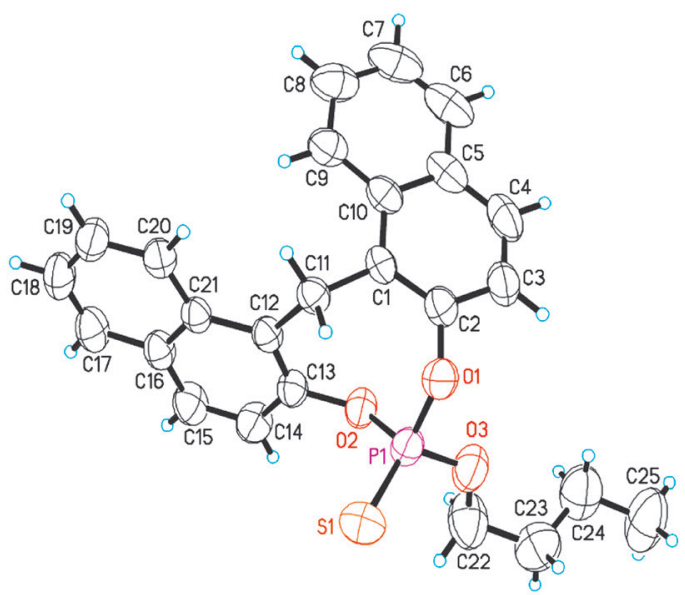

$a$

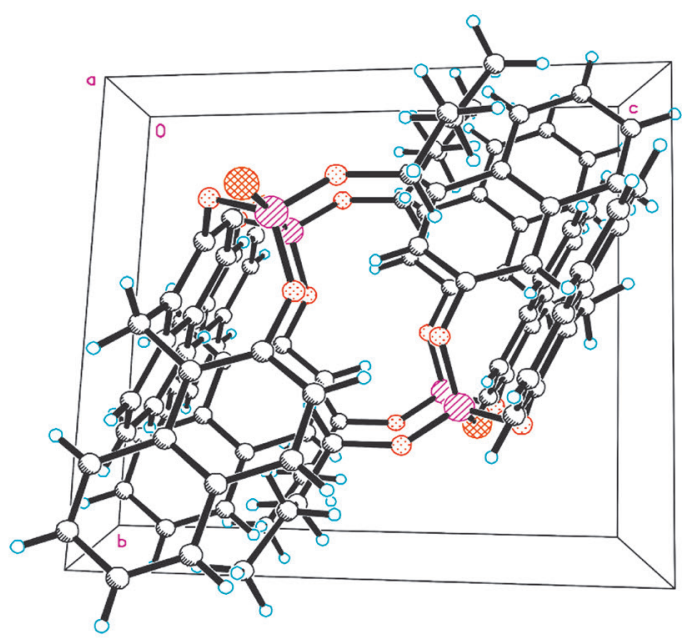

$b$

Figure 1. Molecular structure of 2-thione-2-O-butyl-4,5,7,8-dinaphtho-1,3,2-dioxaphosphacine 8a $(a)$ and fragment of molecules packing in crystal $(b)$.

Table 1. Bond lengths $(\AA)$ and angles for 2-thione-2-O-butyl-4,5,7,8-dinaphtho-1,3,2-dioxaphosphacine 8a.

\begin{tabular}{|c|c|c|c|}
\hline Bond & $d, \AA$ & Angle & $\omega$, grad \\
\hline $\mathrm{P}(1)-\mathrm{O}(1)$ & $1.5824(18)$ & $\mathrm{O}(3)-\mathrm{P}(1)-\mathrm{O}(1)$ & $99.95(10)$ \\
\hline $\mathrm{P}(1)-\mathrm{O}(2)$ & $1.5845(18)$ & $\mathrm{O}(3)-\mathrm{P}(1)-\mathrm{O}(2)$ & $101.68(10)$ \\
\hline $\mathrm{P}(1)-\mathrm{O}(3)$ & $1.5491(19)$ & $\mathrm{O}(1)-\mathrm{P}(1)-\mathrm{O}(2)$ & $105.19(9)$ \\
\hline $\mathrm{P}(1)-\mathrm{S}(1)$ & $1.8993(11)$ & $\mathrm{O}(3)-\mathrm{P}(1)-\mathrm{S}(1)$ & $118.82(9)$ \\
\hline $\mathrm{O}(1)-\mathrm{C}(2)$ & $1.414(3)$ & $\mathrm{O}(1)-\mathrm{P}(1)-\mathrm{S}(1)$ & $112.23(8)$ \\
\hline $\mathrm{O}(2)-\mathrm{C}(13)$ & $1.403(3)$ & $\mathrm{O}(2)-\mathrm{P}(1)-\mathrm{S}(1)$ & $116.79(7)$ \\
\hline $\mathrm{O}(3)-\mathrm{C}(22)$ & $1.422(4)$ & $\mathrm{C}(13)-\mathrm{O}(2)-\mathrm{P}(1)$ & $128.02(14)$ \\
\hline $\mathrm{C}(1)-\mathrm{C}(2)$ & $1.365(3)$ & $\mathrm{C}(22)-\mathrm{O}(3)-\mathrm{P}(1)$ & $124.12(18)$ \\
\hline $\mathrm{C}(1)-\mathrm{C}(11)$ & $1.517(3)$ & $C(2)-C(1)-C(10)$ & $117.8(2)$ \\
\hline$C(2)-C(3)$ & $1.393(3)$ & $C(2)-C(1)-C(11)$ & $119.5(2)$ \\
\hline$C(11)-C(12)$ & $1.521(3)$ & $\mathrm{C}(10)-\mathrm{C}(1)-\mathrm{C}(11)$ & $122.7(2)$ \\
\hline$C(12)-C(13)$ & $1.370(3)$ & $\mathrm{C}(1)-\mathrm{C}(2)-\mathrm{O}(1)$ & $118.3(2)$ \\
\hline$C(13)-C(14)$ & $1.400(3)$ & $\mathrm{C}(3)-\mathrm{C}(2)-\mathrm{O}(1)$ & $117.6(2)$ \\
\hline$C(22)-C(23)$ & $1.509(4)$ & $\mathrm{C}(1)-\mathrm{C}(11)-\mathrm{C}(12)$ & $114.39(17)$ \\
\hline \multirow[t]{7}{*}{$C(23)-C(24)$} & $1.466(5)$ & $\mathrm{C}(13)-\mathrm{C}(12)-\mathrm{C}(21)$ & $117.02(18)$ \\
\hline & & $C(13)-C(12)-C(11)$ & $121.49(18)$ \\
\hline & & $\mathrm{C}(12)-\mathrm{C}(13)-\mathrm{C}(14)$ & $123.7(2)$ \\
\hline & & $\mathrm{C}(12)-\mathrm{C}(13)-\mathrm{O}(2)$ & $122.08(18)$ \\
\hline & & $\mathrm{C}(14)-\mathrm{C}(13)-\mathrm{O}(2)$ & $114.10(19)$ \\
\hline & & $\mathrm{O}(3)-\mathrm{C}(22)-\mathrm{C}(23)$ & $108.3(2)$ \\
\hline & & $C(24)-C(23)-C(22)$ & $114.3(3)$ \\
\hline
\end{tabular}


The observed formation of phosphacines upon phosphorylation of 1 with acyclic amidophosphites has precluded formation of macrocycles containing two fragments of bisnaphthol. Therefore, we have proposed two-step approach to 14-membered macrocyclic systems, ${ }^{[18]}$ containing a fragment of bisnaphthol 1 and a fragment of resorcinol 10 or 1,3-dihydroxynaphthalene 11. This approach implies synthesis of primary bisphosphorylation of diols $\mathbf{1 0}$ and $\mathbf{1 1}$ with hexaethyltriamidophosphite (HETA) 2c with subsequent cyclization of resulting bisphosphorylated derivatives 12 and 13 with bisnaphthol 1 (Scheme 4).

Reactions were performed in 1,4-dioxane. The completion of bisphosphorylation of diols $\mathbf{1 0}$ and $\mathbf{1 1}$ was confirmed by ${ }^{31} \mathrm{P}$ NMR spectroscopy - there was observed a singlet at $\delta_{\mathrm{p}}=132.8 \mathrm{ppm}$, which is characteristic for diamidoesters of phosphorous esters with aromatic radicals $\mathbf{- 1 2}$ and $\mathbf{1 3}$. Then, bisphenol 1 was added to reaction mixture and the mixture was kept for 3 days. During this time the signal at $132.8 \mathrm{ppm}$ gradually vanished and a new signal at $140.5 \mathrm{ppm}$ became intensive. This signal corresponded to the target amidodiesters.

Resulting macrocycles 14 and 15 were found to be unstable. In solution they have rearranged with the formation of 1,3,2-dioxaphosphacine $\mathbf{3 c}$ and uniform cyclophanes. ${ }^{[3,16]}$

However, we have tried to isolate target macrocycles. With this aim the reaction mixtures containing products with $\delta_{\mathrm{P}}=140 \mathrm{ppm}$ were evaporated to minimal volume and cooled resulting in formation of oily product. The solvent was decanted, the oil was washed with cold acetonitrile and dried in vacuo. Their NMR spectra corresponded to the target macrocycles 14 and $\mathbf{1 5}$, however, they also contained resonance signals of 1,3,2-dioxaphosphacine $\mathbf{3 c}$ impurity. Isolation of pure macrocycles was possible only after their conversion into thiones and phosphates.

Sulfurization of cyclophosphites $\mathbf{1 4}$ and $\mathbf{1 5}$ was performed with elemental sulfur in $\mathrm{CH}_{2} \mathrm{Cl}_{2}$ during 1 day. Result- ing cyclo(bisthioneamidophosphates) $\mathbf{1 6}$ and $\mathbf{1 7}$ were isolated by column chromatography in 30 and $25 \%$ yields respectively as viscous oils. Their ${ }^{31} \mathrm{P}$ NMR spectra contained singlets in $68 \mathrm{ppm}$ region, what corresponded to amidoesters of thionephosphoric acid.

Oxidation of cyclophosphites $\mathbf{1 4}$ and $\mathbf{1 5}$ was peformed with urea peroxide in $\mathrm{CH}_{2} \mathrm{Cl}_{2}$ for 1 day. Phosphates $\mathbf{1 8}$ and 19 were isolated by precipitation with hexane. They were isolated as low-melting powders in 79 and $65 \%$ yields respectively. Their ${ }^{31} \mathrm{P}$ NMR spectra contained singlets at $1 \mathrm{ppm}$ which corresponded to monoamidophosphates. ${ }^{1} \mathrm{H}$ NMR spectrum of compound 19 revealed some broadening of resonance signals.

\section{Conclusions}

1. We have investigated phosphorylation of 2,2'-dihydroxy-1,1'-dinaphthylmetane with derivatives of $\mathrm{P}^{\mathrm{III}}-$ hexabutyltriamide, trimorpholinephosphite, tetraethyl- and tetrabutyldiamidophenylphosphites, as well as tetraethyldiamidobutylphosphite.

2. The equiprobable leaving of amide or aromatic ester substituent was found upon formation of 1,3,2-dioxaphosphacine.

3. We have synthesized phosphamacrocyclic systems, containing 2,2'-dihydroxy-1,1'-dinaphthylmethane as a building block, the oxidation reactions of these macrocycles were investigated.

\section{Experimental}

All syntheses were conducted in dry solvents under an argon atmosphere. ${ }^{1} \mathrm{H},{ }^{13} \mathrm{C}$ and ${ }^{31} \mathrm{P}$ NMR spectra were recorded on a JEOL ECX-400 spectrometer operating at $400,100.5$ and $161.8 \mathrm{MHz}$<smiles>CCCCCCNCC</smiles><smiles>[Z16]NP(NCC)O[Al]NP(NCC)NCC</smiles>

12,13<smiles>CCNC(C)(C)[Ge](C)(C)C</smiles><smiles>[Z15]CCCCCNP1O[Al]OP(NCC)Oc2ccc3ccccc3c2Cc2c(ccc3ccccc23)O1</smiles>

14,15<smiles>[X][Y]1(NCC)O[Al]O[PH]([X])(NCC)Oc2ccc3ccccc3c2Cc2c(ccc3ccccc23)O1</smiles>

$16-19$

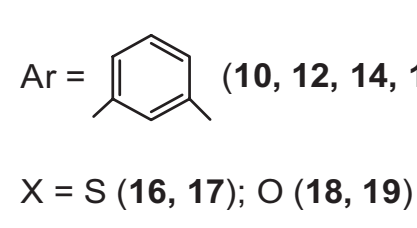<smiles>Cc1cc(C)c2ccccc2c1</smiles>
$(11,13,15,17,19)$

Scheme 4. 
respectively; ${ }^{1} \mathrm{H}$ and ${ }^{13} \mathrm{C}$ NMR spectra were recorded in $\mathrm{CDCl}_{3}$; chemical shifts $(\delta, \mathrm{ppm})$ were referenced to TMS $\left({ }^{1} \mathrm{H}\right.$ and $\left.{ }^{13} \mathrm{C}\right)$ or to $\left.85 \% \mathrm{H}_{3} \mathrm{PO}_{4}{ }^{(31} \mathrm{P}\right)$. Spin-spin coupling constants $(J)$ are given in $\mathrm{Hz}$. Mass spectra were measured on a Bruker Ultraflex MALDI-TOF spectrometer using a nitrogen laser $(\lambda=337 \mathrm{~nm})$ and trihydroxyanthracene as a matrix. Crystals of compound 8a have obtained by crystallization from hexane. X-ray analysis was performed on an automatic CAD-4 Enraf-Nonius diffractometer ( $\beta$-filter, $\lambda($ MoKa) $\theta / 2 \theta$ data collection, $\theta_{\max }=24.88^{\circ}$ ). Colorless triclinic crystal $\left(\mathrm{C}_{25} \mathrm{H}_{23} \mathrm{O}_{3} \mathrm{PS}, M=434.46\right)$, size $0.56 \times 0.40 \times 0.15 \mathrm{~mm}, a=9.881(2)$ $\AA, b=10.846(2) \AA, c=11.482(2) \AA, \alpha=87.10(3)^{\circ}, \beta=74.82(3)^{\circ}$, $\gamma=71.12(3)^{\circ} . V=1122.9(5) \AA^{3} . Z=2 . \rho=1.258 \mathrm{mg} / \mathrm{cm}^{3}$. Number of reflections: 4433, independent reflections: $4172(R(\mathrm{int})=0.0200)$. $F(000)=456 ; R_{1}(F)=0.0431, w R_{2}\left(F^{2}\right)=0.1307$. Refinement method: full-matrix least squares on $F^{2}$ for non-hydrogen atoms. All hydrogen atoms have included in the refinement with fixed parameters (placed in calculated positions) in the isotropic approximation. Crystallographic data reported in this paper have been deposited with Cambridge Crysrallographic Data Centre (№ CCDC 1052567). Column adsorption chromatography was operated on silica gel L 100/250; TLC was performed on Silufol plates (UV-254) using $\mathrm{C}_{6} \mathrm{H}_{14}$ :dioxane, 5:1 (A), $\mathrm{C}_{6} \mathrm{H}_{6}$ :dioxane, $3: 1$ (B). Detection was achieved using iodine vapor treatment and calcination.

2,2'-Dihydroxy-1,1'-dinaphthylmethane 1 was synthesized by the method, ${ }^{[19]}$ HBTA $2 \boldsymbol{a},{ }^{[16]}$ tetraethyl- and tetrabutyldiamidophenylphosphites $(\mathbf{4 b , c )}),{ }^{[15]}$ HETA 12. ${ }^{[20]}$

2-Dibutylamido-4,5, 7,8-dinaphtho-1,3,2-dioxaphosphacine

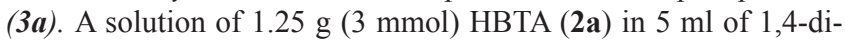
oxane was added to a solution of $0.3 \mathrm{~g}(1 \mathrm{mmol})$ of bisnaphthol 1 in $4 \mathrm{ml}$ of 1,4-dioxane. The reaction mixture was kept at room temperature for $2 \mathrm{~h}$. The solvent was evaporated in vacuo $(12 \mathrm{~mm} \mathrm{Hg})$, and the residue was chromatographed on a column, the resulting product was eluted by hexane:dioxane $(10: 1)$ system. The resulting material was dried in vacuo for $1.5 \mathrm{~h}\left(1 \mathrm{~mm} \mathrm{Hg}, 60^{\circ} \mathrm{C}\right)$. Yield 0.41 $\mathrm{g}(89 \%) . \mathrm{R}_{f} 0.69$ (A). ${ }^{1} \mathrm{H}$ NMR $\delta_{\mathrm{H}}$ ppm: $0.97\left(6 \mathrm{H}, \mathrm{t},{ }^{3} \mathrm{~J}_{\mathrm{HH}}=7.3, \mathrm{CH}_{3}\right)$, $1.40\left(4 \mathrm{H}, \mathrm{m}, \mathrm{CH}_{2}\right), 1.62\left(4 \mathrm{H}, \mathrm{m}, \mathrm{CH}_{2}\right), 3.19\left(4 \mathrm{H}, \mathrm{m},{ }^{3} J_{\mathrm{PH}}=11.0\right.$, $\left.\mathrm{N}-\mathrm{CH}_{2}\right), 4.58\left(1 \mathrm{H}, \mathrm{dd},{ }^{2} \mathrm{~J}_{\mathrm{HH}}=16.0, \mathrm{Ar}-\mathrm{CH}_{2}\right), 5.11\left(1 \mathrm{H}, \mathrm{d},{ }^{2} \mathrm{~J}_{\mathrm{HH}}=16.0\right.$, $\left.\operatorname{Ar}-\mathrm{CH}_{2}\right), 7.18\left(2 \mathrm{H}, \mathrm{d},{ }^{3} \mathrm{~J}_{\mathrm{HH}}=8.7, \mathrm{CH}^{3}\right), 7.38\left(2 \mathrm{H}, \mathrm{dd},{ }^{3} J_{\mathrm{HH}}=6.9 ; 7.8\right.$, $\left.\mathrm{CH}^{7}\right), 7.49\left(2 \mathrm{H}, \mathrm{dd},{ }^{3} \mathrm{JHH}_{\mathrm{HH}}=7.8, \mathrm{CH}^{6}\right), 7.68\left(2 \mathrm{H}, \mathrm{d},{ }^{3} \mathrm{~J}_{\mathrm{HH}}=8.8, \mathrm{CH}^{4}\right)$, $7.80\left(2 \mathrm{H}, \mathrm{d},{ }^{3} \mathrm{~J}_{\mathrm{HH}}=7.8, \mathrm{CH}^{5}\right), 8.22\left(2 \mathrm{H}, \mathrm{d},{ }^{3} \mathrm{~J}_{\mathrm{HH}}=8.7, \mathrm{CH}^{8}\right) .{ }^{13} \mathrm{C} \mathrm{NMR}$ $\delta_{\mathrm{C}}$ ppm: $14.1\left(\mathrm{CH}_{3}\right), 20.2\left(\mathrm{CH}_{2}\right), 25.1\left(\mathrm{Ar}-\mathrm{CH}_{2}\right), 30.9\left(\mathrm{CH}_{2}\right), 43.7$ $\left(\mathrm{d},{ }^{2} J_{\mathrm{PC}}=19.1, \mathrm{CH}_{2}-\mathrm{N}\right), 121.9\left(\mathrm{~d},{ }^{3} J_{\mathrm{PC}}=5.8, \mathrm{C}^{3} \mathrm{H}\right), 122.6\left(\mathrm{~d},{ }^{3} J_{\mathrm{PC}}=3.8\right.$, $\left.\mathrm{C}^{1}\right), 123.4\left(\mathrm{C}^{8} \mathrm{H}\right), 124.1\left(\mathrm{C}^{7} \mathrm{H}\right), 126.8\left(\mathrm{C}^{6} \mathrm{H}\right), 128.4\left(\mathrm{C}^{5} \mathrm{H}\right), 128.9$ $\left(\mathrm{C}^{4} \mathrm{H}\right), 130.9\left(\mathrm{C}^{9}\right), 133.0\left(\mathrm{C}^{10}\right), 151.8(\mathrm{C}-\mathrm{O}) .{ }^{31} \mathrm{P}$ NMR (1,4-dioxane) $\delta_{\mathrm{P}}$ ppm: 140.1 .

2-Morpholino-4,5, 7,8-dinaphtho-1,3,2-dioxaphosphacine

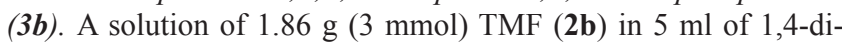
oxane was added to a solution of $0.3 \mathrm{~g}(1 \mathrm{mmol})$ of bisnaphthol 1 in $4 \mathrm{ml}$ of 1,4-dioxane. The reaction mixture was kept at room temperature for $2 \mathrm{~h}$. The solvent was evaporated in vacuo $(12 \mathrm{~mm}$ $\mathrm{Hg}$ ); the residue was added to the $5 \mathrm{ml}$ of acetonitrile. After $12 \mathrm{~h}$ the crystals precipitated were filtered off, washed with hexane and acetonitrile. The resulting material was dried in vacuo for $1.5 \mathrm{~h}(1 \mathrm{~mm}$ $\left.\mathrm{Hg}, 60{ }^{\circ} \mathrm{C}\right)$. Yield $0.37 \mathrm{~g}(90 \%)$. Mp $189-190{ }^{\circ} \mathrm{C} . \mathrm{R}_{f} 0.32(\mathrm{~A}) .{ }^{1} \mathrm{H}$ NMR $\delta_{\mathrm{H}}$ ppm: $3.33\left(4 \mathrm{H}, \mathrm{m},{ }^{3} \mathrm{~J}_{\mathrm{PH}}=7.8,{ }^{3} \mathrm{~J}_{\mathrm{HH}}=4.6, \mathrm{~N}-\mathrm{CH}_{2}\right), 3.75(4 \mathrm{H}$, $\left.\mathrm{dd},{ }^{3} J_{\mathrm{HH}}=4.6, \mathrm{O}-\mathrm{CH}_{2}\right), 4.56\left(1 \mathrm{H}, \mathrm{dd},{ }^{2} J_{\mathrm{HH}}=16.1, \mathrm{Ar}-\mathrm{CH}_{2}\right), 5.09(1 \mathrm{H}$, $\left.\mathrm{d},{ }^{2} \mathrm{JHH}_{\mathrm{HH}}=16.1, \mathrm{Ar}-\mathrm{CH}_{2}\right), 7.18\left(2 \mathrm{H}, \mathrm{d},{ }^{3} \mathrm{~J}_{\mathrm{HH}}=8.7, \mathrm{CH} 3\right), 7.39(2 \mathrm{H}, \mathrm{dd}$, $\left.{ }^{3} J_{\mathrm{HH}}=7.0 ; 7.6, \mathrm{CH}^{7}\right), 7.50\left(2 \mathrm{H}, \mathrm{dd},{ }^{3} J_{\mathrm{HH}}=7.0 ; 8.3, \mathrm{CH}^{6}\right), 7.69(2 \mathrm{H}, \mathrm{d}$, $\left.{ }^{3} J_{\mathrm{HH}}=8.7, \mathrm{CH}^{4}\right), 7.81\left(2 \mathrm{H}, \mathrm{d},{ }^{3} J_{\mathrm{HH}}=8.2, \mathrm{CH}^{5}\right), 8.21\left(2 \mathrm{H}, \mathrm{d},{ }^{3} J_{\mathrm{HH}}=8.7\right.$, $\left.\mathrm{CH}^{8}\right) .{ }^{13} \mathrm{C}$ NMR $\delta_{\mathrm{C}}$ ppm: $25.1\left(\mathrm{Ar}-\mathrm{CH}_{2}\right), 44.2\left(\mathrm{~d},{ }^{2} \mathrm{~J}_{\mathrm{PC}}=17.2, \mathrm{C} H_{2}-\mathrm{N}\right)$, $68.1(\mathrm{CH}-\mathrm{O}), 121.9\left(\mathrm{~d},{ }^{3} J_{\mathrm{PC}}=5.7, \mathrm{C}^{3} \mathrm{H}\right), 123.4\left(\mathrm{C}^{8} \mathrm{H}\right), 124.4\left(\mathrm{C}^{1}\right)$, $126.9\left(\mathrm{C}^{7} \mathrm{H}\right), 128.6\left(\mathrm{C}^{6} \mathrm{H}\right), 128.9\left(\mathrm{C}^{4,5} \mathrm{H}\right), 131.1\left(\mathrm{C}^{9}\right), 132.9\left(\mathrm{C}^{10}\right)$, $151.1(\mathrm{C}-\mathrm{O}) .{ }^{31} \mathrm{P}$ NMR $\left(\mathrm{CH}_{2} \mathrm{Cl}_{2}\right) \delta_{\mathrm{p}} \mathrm{ppm}: 134.3$.

Phosphorylation of 2,2'-dihydroxy-1,1'-dinaphthylmethane 1 with diamidoesters of phosphorous acid 4a-c (general procedure). Solution of diamidophosphite $4 \mathbf{a}-\mathbf{c}$ in $4 \mathrm{ml}$ of acetonitrile or 1,4-dioxane was mixed with solution of $1 \mathrm{mmol}$ of bisnaphthol $\mathbf{1}$ in acetonitrile or dioxane respectively, at room temperature and continuous stirring. Molar ratios of bisnaphthol $\mathbf{1}$ and diamidophosphite 4a-c were 1:1, 1:2 or 1:3. In two weeks sulfur was added to reaction mixtures, its amount corresponded to that of used diamidophosphite. In two days the reaction mixtures were evaporated, the residues were dissolved in minimal volume of benzene and chromatographed eluting products with hexane:dioxane, 10:1 mixture. Resulting compounds were dried in vacuo $\left(1 \mathrm{~mm} \mathrm{Hg}, 70^{\circ} \mathrm{C}\right)$ for 2 hours.

2-Thione-2-dibutylamido-4,5,7,8-dinaphtho-1,3,2-dioxaphosphacine (7a). Yield $0.39 \mathrm{~g}(79 \%)$. M.p. $112-113{ }^{\circ} \mathrm{C} . \mathrm{R}_{f} 0.57$ (A). ${ }^{1} \mathrm{H}$ NMR $\delta_{\mathrm{H}}$ ppm: $0.97\left(6 \mathrm{H}, \mathrm{t},{ }^{3} \mathrm{~J}_{\mathrm{HH}}=7.8, \mathrm{CH}_{3}\right), 1.40(4 \mathrm{H}, \mathrm{m}$, $\left.\mathrm{CH}_{2}\right), 1.70\left(4 \mathrm{H}, \mathrm{m}, \mathrm{CH}_{2}\right), 3.35\left(4 \mathrm{H}, \mathrm{m},{ }^{3} J_{\mathrm{PH}}=7.3, \mathrm{~N}-\mathrm{CH}_{2}\right), 4.99$ $\left(1 \mathrm{H}, \mathrm{d},{ }^{2} J_{\mathrm{HH}}=16.0, \mathrm{Ar}-\mathrm{CH}_{2}\right), 5.12\left(1 \mathrm{H}, \mathrm{d},{ }^{2} J_{\mathrm{HH}}=15.6, \mathrm{Ar}-\mathrm{CH}_{2}\right), 7.19$ $\left(2 \mathrm{H}, \mathrm{d},{ }^{3} J_{\mathrm{HH}}=9.2, \mathrm{CH}^{3}\right), 7.44\left(2 \mathrm{H}, \mathrm{dd},{ }^{3} J_{\mathrm{HH}}=7.3, \mathrm{CH}^{7}\right), 7.55(2 \mathrm{H}$, $\left.\mathrm{dd},{ }^{3} \mathrm{H}_{\mathrm{HH}}=7.3 ; 8.3, \mathrm{CH}^{6}\right), 7.73\left(2 \mathrm{H}, \mathrm{d},{ }^{3} J_{\mathrm{HH}}=8.7, \mathrm{CH}^{4}\right), 7.83(2 \mathrm{H}, \mathrm{d}$ $\left.{ }^{3} J_{\mathrm{HH}}=7.3, \mathrm{CH}^{5}\right), 8.34\left(2 \mathrm{H}, \mathrm{d},{ }^{3} \mathrm{JHH}_{\mathrm{HH}}=8.7, \mathrm{CH}^{8}\right) .{ }^{13} \mathrm{C} \mathrm{NMR}_{\mathrm{C}}$ ppm: 14.1 $\left(\mathrm{CH}_{3}\right), 20.2\left(\mathrm{CH}_{2}\right), 25.1\left(\mathrm{Ar}-\mathrm{CH}_{2}\right), 30.7\left(\mathrm{CH}_{2}\right), 46.7\left(\mathrm{CH}_{2}-\mathrm{N}\right), 121.9$ $\left(\mathrm{C}^{3} \mathrm{H}\right), 123.8\left(\mathrm{C}^{1}\right), 124.9\left(\mathrm{C}^{8} \mathrm{H}\right), 125.1\left(\mathrm{C}^{7} \mathrm{H}\right), 127.1\left(\mathrm{C}^{6} \mathrm{H}\right), 128.4$ $\left(\mathrm{C}^{5} \mathrm{H}\right), 128.9\left(\mathrm{C}^{4} \mathrm{H}\right), 131.8\left(\mathrm{C}^{9}\right), 132.9\left(\mathrm{C}^{10}\right), 149.9\left(\mathrm{~d},{ }^{2} J_{\mathrm{PC}}=11.5\right.$, C-O). ${ }^{31}$ P NMR (1,4-dioxane) $\delta_{\mathrm{p}}$ ppm: 67.8 .

2-Thione-2-O-butyl-4,5, 7,8-dinaphtho-1,3,2-dioxaphosphacine (8a). Yield $0.087 \mathrm{~g}(20 \%)$. M.p. $142-143{ }^{\circ} \mathrm{C} . \mathrm{R}_{f} 0.73$ (A). ${ }^{1} \mathrm{H}$ NMR $\delta_{\mathrm{H}}$ ppm: $0.98\left(3 \mathrm{H}, \mathrm{t},{ }^{3} J_{\mathrm{HH}}=7.3, \mathrm{CH}_{3}\right), 1.50\left(2 \mathrm{H}, \mathrm{m}, \mathrm{CH}_{2}\right)$, $1.80\left(2 \mathrm{H}, \mathrm{m},{ }^{3} J_{\mathrm{HH}}=6.4 ; 7.8, \mathrm{CH}_{2}\right), 4.36\left(2 \mathrm{H}, \mathrm{m},{ }^{3} \mathrm{~J}_{\mathrm{PH}}=12.8, \mathrm{O}-\mathrm{CH}_{2}\right)$, $4.76\left(1 \mathrm{H}, \mathrm{d},{ }^{2} J_{\mathrm{HH}}=16.0, \mathrm{Ar}-\mathrm{CH}_{2}\right), 5.18\left(1 \mathrm{H}, \mathrm{d},{ }^{2} \mathrm{~J}_{\mathrm{HH}}=16.0, \mathrm{Ar}-\mathrm{CH}_{2}\right)$, $7.22\left(2 \mathrm{H}, \mathrm{d},{ }^{3} \mathrm{~J}_{\mathrm{HH}}=8.9,{ }^{4} J_{\mathrm{PH}}=1.4, \mathrm{CH}^{3}\right), 7.47\left(\mathrm{dd}, 2 \mathrm{H},{ }^{3} J_{\mathrm{HH}}=7.3\right.$, $\left.\mathrm{CH}^{7}\right), 7.57\left(2 \mathrm{H}, \mathrm{dd},{ }^{3} \mathrm{H}_{\mathrm{HH}}=7.8, \mathrm{CH}^{6}\right), 7.77\left(2 \mathrm{H}, \mathrm{d},{ }^{3} \mathrm{~J}_{\mathrm{HH}}=8.8, \mathrm{CH}^{4}\right)$, $7.86\left(2 \mathrm{H}, \mathrm{d},{ }^{3} J_{\mathrm{HH}}=8.2, \mathrm{CH}^{5}\right), 8.28\left(2 \mathrm{H}, \mathrm{d},{ }^{3} J_{\mathrm{HH}}=8.3, \mathrm{CH}^{8}\right),{ }^{13} \mathrm{C} \mathrm{NMR}$ $\delta_{\mathrm{C}}$ ppm: $13.8\left(\mathrm{CH}_{3}\right), 24.4\left(\mathrm{Ar}-\mathrm{CH}_{2}\right), 32.2\left(\mathrm{CH}_{2}\right), 69.5\left(\mathrm{CH}_{2}-\mathrm{O}\right)$, $120.9\left(\mathrm{C}^{3} \mathrm{H}\right), 123.6\left(\mathrm{C}^{8} \mathrm{H}\right), 124.6\left(\mathrm{~d}, \mathrm{C}^{1}\right), 125.5\left(\mathrm{C}^{7} \mathrm{H}\right), 127.4\left(\mathrm{C}^{6} \mathrm{H}\right)$, $129.1\left(\mathrm{C}^{4,5} \mathrm{H}\right), 132.1\left(\mathrm{C}^{9}\right), 132.8\left(\mathrm{C}^{10}\right), 148.9(\mathrm{~d}, \mathrm{C}-\mathrm{O}) .{ }^{31} \mathrm{P}$ NMR (1,4-dioxane) $\delta_{\mathrm{p}}$ ppm: 59.5 .

2-Thione-2-O-phenyl-4,5, 7,8-dinaphtho-1,3,2-dioxaphosphacine (8b). Yield $0.068 \mathrm{~g}(15 \%)$. M.p. $158-159^{\circ} \mathrm{C} . \mathrm{R}_{f} 0.51$ (A) ${ }^{1} \mathrm{H}$ NMR $\delta_{\mathrm{H}}$ ppm: $4.80\left(1 \mathrm{H}, \mathrm{d},{ }^{2} J_{\mathrm{HH}}=16.0, \mathrm{Ar}-\mathrm{CH}_{2}\right), 5.24(1 \mathrm{H}, \mathrm{d}$, $\left.{ }^{2} J_{\mathrm{HH}}=16.0, \mathrm{Ar}-\mathrm{CH}_{2}\right), 7.20\left(2 \mathrm{H}, \mathrm{d},{ }^{3} J_{\mathrm{HH}}=8.8, \mathrm{CH}^{3}\right), 7.27(2 \mathrm{H}, \mathrm{d}, \mathrm{Ph})$, 7.36-7.44 (3H, m, Ph), $7.49\left(2 \mathrm{H}, \mathrm{dd},{ }^{3} \mathrm{H}_{\mathrm{HH}}=7.3 ; 7.8, \mathrm{CH}^{7}\right), 7.58$ $\left(2 \mathrm{H}, \mathrm{dd},{ }^{3} \mathrm{~J}_{\mathrm{HH}}=7.3 ; 7.8, \mathrm{CH}^{6}\right), 7.75\left(2 \mathrm{H}, \mathrm{d},{ }^{3} \mathrm{~J}_{\mathrm{HH}}=9.0, \mathrm{CH}^{4}\right), 7.86$ $\left(2 \mathrm{H}, \mathrm{d},{ }^{3} J_{\mathrm{HH}}=8.3, \mathrm{CH}^{5}\right), 8.29\left(2 \mathrm{H}, \mathrm{d},{ }^{3} J_{\mathrm{HH}}=8.7, \mathrm{CH}^{8}\right) .{ }^{13} \mathrm{C} \mathrm{NMR} \delta_{\mathrm{c}}$ ppm: $24.4\left(\mathrm{Ar}^{-\mathrm{CH}_{2}}\right), 120.8\left(\mathrm{C}^{3} \mathrm{H}\right), 121.2(o-\mathrm{Ph}), 123.6\left(\mathrm{C}^{8} \mathrm{H}\right), 124.5$ $\left(\mathrm{d},{ }^{3} J_{\mathrm{PC}}=7.7, \mathrm{C}^{1}\right), 125.6\left(\mathrm{C}^{7} \mathrm{H}\right), 125.9(p-\mathrm{Ph}), 127.5\left(\mathrm{C}^{6} \mathrm{H}\right), 129.2$ $\left(\mathrm{C}^{4,5} \mathrm{H}\right), 129.9(m-\mathrm{Ph}), 133.2\left(\mathrm{C}^{9}\right), 132.8\left(\mathrm{C}^{10}\right), 150.1(\mathrm{~d}, \mathrm{C}-\mathrm{O}), 153.2$ (d, Ph-O). ${ }^{31} \mathrm{P}$ NMR (1,4-dioxane) $\delta_{\mathrm{p}}$ ppm: 53.5. Mass spectrum (MALDI) $m / z: 455.48[M+\mathrm{H}]^{+}$.

2,2 '-Bis(diethylamidophenylthionephosphatoxy)-1,1'-dinaphthylmethane (9b). Yield 0.06 g (8\%). M.p. $157-158^{\circ} \mathrm{C} . \mathrm{R}$ $0.63(\mathrm{~A}) .{ }^{1} \mathrm{H}$ NMR $\delta_{\mathrm{H}}$ ppm: $1.17\left(6 \mathrm{H}, \mathrm{t},{ }^{3} \mathrm{~J}_{\mathrm{HH}}=7.3, \mathrm{CH}_{3}\right), 1.32(6 \mathrm{H}$, $\left.\mathrm{t},{ }^{3} \mathrm{~J}_{\mathrm{HH}}=7.0, \mathrm{CH}_{3}\right), 3.46\left(8 \mathrm{H}, \mathrm{m},{ }^{3} \mathrm{~J}_{\mathrm{PH}}=14.3, \mathrm{CH}_{2}\right), 5.00(1 \mathrm{H}, \mathrm{d}$, $\left.{ }^{2} J_{\mathrm{HH}}=15.7, \mathrm{Ar}-\mathrm{CH}_{2}\right), 5.14\left(1 \mathrm{H}, \mathrm{d},{ }^{2} J_{\mathrm{HH}}=15.7, \mathrm{Ar}-\mathrm{CH}_{2}\right), 7.19(4 \mathrm{H}, \mathrm{d}$, $\mathrm{Ph}), 7.24\left(2 \mathrm{H}, \mathrm{d}, \mathrm{CH}^{3}\right), 7.32\left(4 \mathrm{H}, \mathrm{t},{ }^{3} J_{\mathrm{HH}}=8.0, \mathrm{Ph}\right), 7.36(2 \mathrm{H}, \mathrm{m}, \mathrm{Ph})$, $7.49\left(2 \mathrm{H}, \mathrm{dd},{ }^{3} J_{\mathrm{HH}}=8.0, \mathrm{CH}^{7}\right), 7.56\left(2 \mathrm{H}, \mathrm{dd},{ }^{3} J_{\mathrm{HH}}=8.0,{ }^{4} J_{\mathrm{HH}}=1.1\right.$, $\left.\mathrm{CH}^{6}\right), 7.74\left(2 \mathrm{H}, \mathrm{d},{ }^{3} J_{\mathrm{HH}}=8.8, \mathrm{CH}^{4}\right), 7.86\left(2 \mathrm{H}, \mathrm{d},{ }^{3} J_{\mathrm{HH}}=7.7, \mathrm{CH}^{5}\right)$, $8.35\left(2 \mathrm{H}, \mathrm{d},{ }^{3} \mathrm{~J}_{\mathrm{HH}}=8.8, \mathrm{CH}^{8}\right) .{ }^{31} \mathrm{P}$ NMR $\left(\mathrm{CH}_{3} \mathrm{CN}\right) \delta_{\mathrm{p}} \mathrm{ppm}: 66.8$. Found, \%: $\mathrm{P} 8.18 . \mathrm{C}_{41} \mathrm{H}_{44} \mathrm{~N}_{2} \mathrm{O}_{4} \mathrm{P}_{2} \mathrm{~S}_{2}$. Calculated, \%: P 8.21.

Cyclophosphorylation of 2,2'-dihydroxy-1,1'-dinaphthylmethane (general procedure). A solution $0.16 \mathrm{~g}(1 \mathrm{mmol})$ of dihydroxynaphthalene $\mathbf{1 0}$ or $\mathbf{1 1}$ in $6 \mathrm{ml}$ of 1,4-dioxane was added at stirring at room temperature to $0.5 \mathrm{~g}(2 \mathrm{mmol})$ HETA $2 \mathrm{c}$. After $12 \mathrm{~h}$ (10) or $6 \mathrm{~h}(\mathbf{1 1})$ a solution of $0.3 \mathrm{~g}(1 \mathrm{mmol})$ of bisnaphthol 1 in $4 \mathrm{ml}$ of 1,4-dioxane was added to the reaction mixture. The mixture was stirred for $5 \mathrm{~h}$ and left for $60 \mathrm{~h}$. The solvent was evaporated in vacuo $(12 \mathrm{~mm} \mathrm{Hg})$; the remaining oily precipitate was washed twice with cold acetonitrile and dried in vacuo for $2 \mathrm{~h}\left(1 \mathrm{~mm} \mathrm{Hg}, 60^{\circ} \mathrm{C}\right)$.

1,3(1,2)-Dinaphthalina-7(1,3)-benzena-4, 6, 8, 10-tetraoxa-5,9-di(diethylamidate)phosphacyclodeca-phane (14). Yield $0.14 \mathrm{~g}(25 \%)$. Oily substance. $\mathrm{R}_{f} 0.80$ (B). ${ }^{1} \mathrm{H}$ NMR $\delta_{\mathrm{H}}$ ppm: 1.19 $\left(6 \mathrm{H}, \mathrm{t},{ }^{3} J_{\mathrm{HH}}=7.0, \mathrm{CH}_{3}\right), 1.25\left(6 \mathrm{H}, \mathrm{t},{ }^{3} J_{\mathrm{HH}}=7.0, \mathrm{CH}_{3}\right), 3.31(8 \mathrm{H}, \mathrm{m}$, $\left.{ }^{3} J_{\mathrm{PH}}=11.0, \mathrm{~N}-\mathrm{CH}_{2}\right), 4.55\left(1 \mathrm{H}, \mathrm{d},{ }^{2} J_{\mathrm{HH}}=16.2, \mathrm{Ar}-\mathrm{CH}_{2}\right), 5.26(1 \mathrm{H}, \mathrm{d}$, 
$\left.{ }^{2} J_{\mathrm{HH}}=16.2, \mathrm{Ar}-\mathrm{CH}_{2}\right), 6.38\left(2 \mathrm{H}, \mathrm{dd},{ }^{3} \mathrm{~J}_{\mathrm{HH}}=7.9,{ }^{4} \mathrm{~J}_{\mathrm{HH}}=2.3, o-\mathrm{CH}\right), 6.79$ $\left(1 \mathrm{H}, \mathrm{s}, o^{\prime}-\mathrm{CH}\right), 6.95(1 \mathrm{H}, \mathrm{d}, m-\mathrm{CH}), 7.23\left(2 \mathrm{H}, \mathrm{d},{ }^{3} J_{\mathrm{HH}}=8.9, \mathrm{CH}^{3}\right)$, $7.41\left(2 \mathrm{H}, \mathrm{m},{ }^{3} \mathrm{H}_{\mathrm{HH}}=7.5,7.6, \mathrm{CH}^{6}\right), 7.53\left(2 \mathrm{H}, \mathrm{m},{ }^{3} \mathrm{~J}_{\mathrm{HH}}=7.2,7.8, \mathrm{CH}^{7}\right)$, $7.80\left(2 \mathrm{H}, \mathrm{d},{ }^{3} \mathrm{~J}_{\mathrm{HH}}=8.9, \mathrm{CH}^{4}\right), 7.88\left(2 \mathrm{H}, \mathrm{d},{ }^{3} \mathrm{~J}_{\mathrm{HH}}=7.6, \mathrm{CH}^{5}\right), 8.36(2 \mathrm{H}$, $\left.\mathrm{d},{ }^{3} J_{\mathrm{HH}}=8.2, \mathrm{CH}^{8}\right) .{ }^{31} \mathrm{P}$ NMR (1,4-dioxane) $\delta_{\mathrm{p}}$ ppm: 140.8 .

Thionephosphoramidates $(\mathbf{1 6}, 17)$ (general procedure). Sulfur $0.064 \mathrm{~g}(2 \mathrm{mmol})$ was added to cyclophosphite $(\mathbf{1 4}, \mathbf{1 5})$ in 5 $\mathrm{ml}$ of dichloromethane. The mixture was stirred for $3 \mathrm{~h}$ at room temperature and left for $24 \mathrm{~h}$. The solution was filtered, the solvent was evaporated in vacuo $(12 \mathrm{~mm} \mathrm{Hg})$, and the residue was chromatographed on a column, the resulting product was eluted by the benzene:dioxane $(7: 1)$ system. The resulting material was dried in vacuo for $2 \mathrm{~h}\left(1 \mathrm{~mm} \mathrm{Hg}, 70^{\circ} \mathrm{C}\right)$.

1,3(1,2)-Dinaphtholina-7(1,3)-benzena-4,6,8,10-tetraoxa5,9-di(diethylamidate)thionphosphacyclodeca-phane (17). Yield $0.20 \mathrm{~g}(30 \%)$. Oily substance. $\mathrm{R}_{f} 0.61$ (A). ${ }^{1} \mathrm{H}$ NMR $\delta_{\mathrm{H}} \mathrm{ppm:} 1.00$ $\left(9 \mathrm{H}, \mathrm{t}, \mathrm{CH}_{3}\right), 1.02\left(3 \mathrm{H}, \mathrm{t},{ }^{3} \mathrm{~J}_{\mathrm{HH}}=7.2, \mathrm{CH}_{3}\right), 3.32\left(8 \mathrm{H}, \mathrm{m},{ }^{3} \mathrm{~J}_{\mathrm{PH}}=13.9\right.$, $\left.\mathrm{N}-\mathrm{CH}_{2}\right), 4.70\left(1 \mathrm{H}, \mathrm{d},{ }^{2} J_{\mathrm{HH}}=16.1, \mathrm{Ar}-\mathrm{CH}_{2}\right), 5.14\left(1 \mathrm{H}, \mathrm{d},{ }^{2} J_{\mathrm{HH}}=16.8\right.$, $\left.\mathrm{Ar}-\mathrm{CH}_{2}\right), 6.68\left(2 \mathrm{H}, \mathrm{d},{ }^{3} J_{\mathrm{HH}}=8.0, o-\mathrm{CH}\right), 6.79\left(1 \mathrm{H}, \mathrm{d},{ }^{4} J_{\mathrm{PH}}=4.0\right.$, $\left.o^{\prime}-\mathrm{CH}\right), 6.85\left(1 \mathrm{H}, \mathrm{m},{ }^{3} J_{\mathrm{HH}}=8.0, m-\mathrm{CH}\right), 7.12\left(2 \mathrm{H}, \mathrm{dd},{ }^{3} J_{\mathrm{HH}}=8.8\right.$, $\left.{ }^{4} J_{\mathrm{PH}}=1.5, \mathrm{CH}^{3}\right), 7.38\left(2 \mathrm{H}, \mathrm{dd},{ }^{3} J_{\mathrm{HH}}=7.3,7.8, \mathrm{CH}^{7}\right), 7.48(2 \mathrm{H}, \mathrm{dd}$, $\left.{ }^{3} J_{\mathrm{HH}}=7.7,{ }^{4} J_{\mathrm{HH}}=1.5, \mathrm{CH}^{6}\right), 7.66\left(2 \mathrm{H}, \mathrm{d},{ }^{3} J_{\mathrm{HH}}=8.8, \mathrm{CH}^{4}\right), 7.76(2 \mathrm{H}$, dd, $\left.{ }^{3} J_{\mathrm{HH}}=8.0,{ }^{4} J_{\mathrm{HH}}=1.2, \mathrm{CH}^{5}\right), 8.19\left(2 \mathrm{H}, \mathrm{d},{ }^{3} J_{\mathrm{HH}}=8.0, \mathrm{CH}^{8}\right) .{ }^{31} \mathrm{P}$ NMR (1,4-dioxane) $\delta_{\mathrm{p}}$ ppm: 66.8. Found, \%: C, 62.07; H, 5.69; N, 4.12; P, 9.24. $\mathrm{C}_{35} \mathrm{H}_{38} \mathrm{~N}_{2} \mathrm{O}_{4} \mathrm{P}_{2} \mathrm{~S}_{2}$. Calculated, \%: C, 62.12; H, 5.66; N, $4.14 ; \mathrm{P}, 9.15$.

1,3(1,2), 7(1,3)-Trinaphthalina-4,6,8,10-tetraoxa-5,9-di(diethylamidate) thionphosphacyclodecaphane (18). Yield $0.18 \mathrm{~g}$ (25\%). Oily substance. $\mathrm{R}_{f} 0.58\left(\mathrm{CHCl}_{3}\right) .{ }^{1} \mathrm{H}$ NMR $\delta_{\mathrm{H}}$ ppm: 1.17 $\left(12 \mathrm{H}, \mathrm{t},{ }^{3} \mathrm{~J}_{\mathrm{HH}}=6.9, \mathrm{CH}_{3}\right), 3.26\left(8 \mathrm{H}, \mathrm{m},{ }^{3} \mathrm{~J}_{\mathrm{PH}}=12.4, \mathrm{~N}-\mathrm{CH}_{2}\right), 4.81(1 \mathrm{H}$, $\left.\mathrm{d},{ }^{2} J_{\mathrm{HH}}=16.1, \mathrm{Ar}-\mathrm{CH}_{2}\right), 5.26\left(1 \mathrm{H}, \mathrm{d},{ }^{2} J_{\mathrm{HH}}=15.4, \mathrm{Ar}-\mathrm{CH}_{2}\right), 7.04(1 \mathrm{H}$, $\left.\mathrm{s}, \mathrm{CH}^{2}\right), 7.13\left(2 \mathrm{H}, \mathrm{dd},{ }^{3} \mathrm{JH}_{\mathrm{HH}}=8.8,{ }^{4} \mathrm{~J}_{\mathrm{HH}}=1.1, \mathrm{CH}^{3}\right), 7.22\left(1 \mathrm{H}, \mathrm{s}, \mathrm{CH}^{4}\right)$, $7.25\left(1 \mathrm{H}, \mathrm{dd}, \mathrm{CH}^{6}\right), 7.35\left(1 \mathrm{H}, \mathrm{dd}, \mathrm{CH}^{7}\right), 7.48\left(2 \mathrm{H}, \mathrm{dd}, \mathrm{CH}^{6}\right), 7.59$ $\left(2 \mathrm{H}, \mathrm{dd}, \mathrm{CH}^{7}\right), 7.67\left(2 \mathrm{H}, \mathrm{d},{ }^{3} J_{\mathrm{HH}}=8.8, \mathrm{CH}^{4}\right), 7.74\left(1 \mathrm{H}, \mathrm{d},{ }^{3} \mathrm{~J}_{\mathrm{HH}}=8.8\right.$, $\left.\mathrm{CH}^{5^{\prime}}\right), 7.83\left(2 \mathrm{H}, \mathrm{d},{ }^{3} \mathrm{~J}_{\mathrm{HH}}=8.4, \mathrm{CH}^{5}\right), 8.12\left(1 \mathrm{H}, \mathrm{d},{ }^{3} \mathrm{~J}_{\mathrm{HH}}=8.0, \mathrm{CH}^{8}\right)$, $8.20\left(2 \mathrm{H}, \mathrm{d},{ }^{3} J_{\mathrm{HH}}=8.4, \mathrm{CH}^{8}\right) .{ }^{31} \mathrm{P}$ NMR $\left(1,4\right.$-dioxane) $\delta_{\mathrm{p}} \mathrm{ppm}: 66.4$, 67.0. Found, \%: C, 64.34; H, 5.74; N, 3.89. $\mathrm{C}_{39} \mathrm{H}_{40} \mathrm{~N}_{2} \mathrm{O}_{4} \mathrm{P}_{2} \mathrm{~S}_{2}$. Calculated, \%: C, 64.45; H, 5.55; N, 3.85 .

Phosphoramidates $(\mathbf{1 8}, 19)$ (general procedure). The urea peroxide (commercial hydroperite) $0.23 \mathrm{~g}$ was added to cyclophosphite $(\mathbf{1 4}, \mathbf{1 5})$ in $6 \mathrm{ml}$ of dichloromethane. The mixture was left for $24 \mathrm{~h}$ at room temperature. Then the solution was cooled to $0{ }^{\circ} \mathrm{C}$ and filtered, the solvent was removed in vacuo $(12 \mathrm{~mm} \mathrm{Hg})$ down to a small volume, and $10 \mathrm{ml}$ hexane was added; after 10 min the solution was decanted from precipitate. The procedure was repeated twice. The resulting material was dried in vacuo for $2 \mathrm{~h}$ $\left(1 \mathrm{~mm} \mathrm{Hg}, 60^{\circ} \mathrm{C}\right)$.

1,3(1,2)-Dinaphtholina-7(1,3)-benzena-4,6,8,10-tetraoxa5,9-di(diethylamidate)oxophosphacyclodeca-phane (18). Yield 0.51 g (79 \%). M.p. $89-91{ }^{\circ} \mathrm{C} . \mathrm{R}_{f} 0.63$ (B). ${ }^{1} \mathrm{H}$ NMR $\delta_{\mathrm{H}}$ ppm: 1.03 $\left(9 \mathrm{H}, \mathrm{t}, \mathrm{CH}_{3}\right), 1.30\left(3 \mathrm{H}, \mathrm{t},{ }^{3} \mathrm{~J}_{\mathrm{HH}}=7.3, \mathrm{CH}_{3}\right), 3.18\left(6 \mathrm{H}, \mathrm{m},{ }^{3} \mathrm{~J}_{\mathrm{PH}}=12.4\right.$, $\left.\mathrm{N}-\mathrm{CH}_{2}\right), 3.33\left(2 \mathrm{H}, \mathrm{m}^{3} \mathrm{~J}_{\mathrm{PH}}=12.8, \mathrm{~N}-\mathrm{CH}_{2}\right), 4.93\left(1 \mathrm{H}, \mathrm{d},{ }^{2} \mathrm{~J}_{\mathrm{HH}}=16.2\right.$, Ar- $\left.\mathrm{CH}_{2}\right), 5.16\left(1 \mathrm{H}, \mathrm{d},{ }^{2} J_{\mathrm{HH}}=16.2, \mathrm{Ar}-\mathrm{CH}_{2}\right), 6.58\left(2 \mathrm{H}, \mathrm{d},{ }^{3} \mathrm{~J}_{\mathrm{HH}}=7.7\right.$, $o-\mathrm{CH}), 6.90\left(1 \mathrm{H}, \mathrm{s}, o^{\prime}-\mathrm{CH}\right), 7.05(1 \mathrm{H}, \mathrm{t}, m-\mathrm{CH}), 7.21(2 \mathrm{H}, \mathrm{d}$, $\left.{ }^{3} J_{\mathrm{HH}}=8.5, \mathrm{CH}^{3}\right), 7.43\left(2 \mathrm{H}, \mathrm{dd},{ }^{3} J_{\mathrm{HH}}=6.8,8.1, \mathrm{CH}^{6}\right), 7.54(2 \mathrm{H}, \mathrm{dd}$, $\left.{ }^{3} J_{\mathrm{HH}}=7.7, \mathrm{CH}^{7}\right), 7.73\left(2 \mathrm{H}, \mathrm{d},{ }^{3} J_{\mathrm{HH}}=8.9, \mathrm{CH}^{4}\right), 7.82\left(2 \mathrm{H}, \mathrm{d},{ }^{3} \mathrm{~J}_{\mathrm{HH}}=8.1\right.$,
$\left.\mathrm{CH}^{5}\right), 8.28\left(2 \mathrm{H}, \mathrm{d},{ }^{3} J_{\mathrm{HH}}=8.5, \mathrm{CH}^{8}\right) .{ }^{31} \mathrm{P} \mathrm{NMR}\left(\mathrm{CH}_{2} \mathrm{Cl}_{2}\right) \delta_{\mathrm{P}}$ ppm: 0.9. Mass spectrum (MALDI) $\mathrm{m} / z: 645.22[M+\mathrm{H}]^{+}$.

1,3(1,2), 7(1,3)-Trinaphthalina-4, 6,8,10-tetraoxa-5,9di(diethylamidate)oxophosphacyclodecaphane (19). Yield $0.48 \mathrm{~g}$ (81 \%). M.p. $98-100{ }^{\circ} \mathrm{C} . \mathrm{R}_{f} 0.68$ (B). ${ }^{31} \mathrm{P} \mathrm{NMR}\left(\mathrm{CH}_{2} \mathrm{Cl}_{2}\right) \delta_{\mathrm{P}} \mathrm{ppm}$ : $1.1,0.9$.

Acknowledgements. This work was supported by the Ministry of Education and Science of Russian Federation in the framework of state task for Institution of Higher Education.

\section{References}

1. Nifantyev E.E., Slitikov P.V., Rasadkina E.N. Russ. Chem. Rev. 2007, 76, 327-338.

2. Knyazeva I.R., Burilov A.R., Pudovik M.A., Khabikher V.D. Russ. Chem. Rev. 2013, 82, 150-186.

3. Slitikov P.V., Evdokimenkova Yu.B., Rasadkina E.N., Vasyanina L.K., Nifantiev E.E. Macroheterocycles 2011, 4, 311-323.

4. Slitikov P.V., Rasadkina E.N., Vasyanina L.K., Nifantiev E.E. Macroheterocycles 2013, 6, 170-179.

5. Slitikov P.V., Rasadkina E.N., Vasyanina L.K., Nifantiev E.E. Russ. Chem. Bull. 2013, 62, 2023-2031.

6. Slitikov P.V. Vestnik MGTU im. Baumana, Ser. Estestv. Nauki [Herald of BMSTU, Ser. Natural Sci.] 2014, 94-105 (in Russ.)

7. Steed W.J., Atwood J.L. Supramolecular Chemistry. John Wiley \& Sons, Ltd, 2000. 772 p.

8. Nifant'ev E.E., Rasadkina E.N., Batalova T.A., Bekker A.R., Stash A.I., Belskii V.K. Russ. J. Gen. Chem. 1996, 66, 10811086.

9. Batalova T.A., Rasadkina E.N., Vasyanina L.K., Stash A.I., Belskii V.K., Nifant'ev E.E. Russ. J. Gen. Chem. 1998, 68, 1570-1579.

10. Arshinona R.P. Russ. Chem. Rev. 1988, 57, 1142-1161.

11. Arshinona R.P. Phosphorus, Sulfur, Silicon Relat. Elem. 1992, 68, 155-191.

12. Danilova O.I., Arshinona R.P. Russ. J. Gen. Chem. 1987, 57, 1237-1243.

13. Slitikov P.V., Rasadkina E.N., Vasyanina L.K., Levina I.I., Nifantiev E.E. Russ. J. Gen. Chem. 2014, 84, 505-510.

14. Nifantyev E.E., Rasadkina E.N., Slitikov P.V., Vasyanina L.K. Phosphorus, Sulfur, Silicon Relat. Elem. 2005, 180, 513-526.

15. Nifantyev E.E., Rasadkina E.N., Slitikov P.V., Vasyanina L.K. Phosphorus, Sulfur, Silicon Relat. Elem 2003, 178, 2465-2477.

16. Slitikov P.V., Rasadkina E.N., Korunova Ya.A., Vasyanina L.K., Nifantiev E.E. Russ. J. Gen. Chem. 2010, 80, 920-926.

17. Danilova O.I., Arshinona R.P., Ovodova O.V., Arbuzov B.A. J. Gen. Chem. USSR in Eng. Transl. 1987, 57, 2665-2672.

18. Rasadkina E.N., Slitikov P.V., Mel'nik M.S., Nifant'ev E.E. Russ. Chem. Bull. 2004, 52, 376-382.

19. Abel J. Ber. Dtsch. Chem. Ges. 1892, 25, 3477-3484.

20. Noth H., Vetter H. J. Chem. Ber. 1965, 98, 1981-1987.

Received 24.04.2015

Received revised 18.08.2015

Accepted 20.08.2015 